\title{
Additive Benefits of $\beta$-Alanine Supplementation and Sprint-Interval Training
}

\author{
PHILLIP M. BELLINGER ${ }^{1}$ and CLARE L. MINAHAN ${ }^{1,2}$
}

${ }^{1}$ Griffith University Sport Science, School of Allied Health Sciences, Griffith University, Gold

Coast, Queensland, Australia; ${ }^{2}$ Menzies Health Institute Queensland, Griffith University, Gold Coast, Queensland, Australia.

\section{Correspondence:}

Phillip Bellinger

Griffith University Sport Science

School of Allied Health Sciences

Griffith University, 4222

Queensland, Australia

Phone: (617) $55529219 \quad$ Fax: (617) $55528674 \quad$ Email: p.bellinger@griffith.edu.au

The authors have no financial relationships relevant to this article to disclose and no competing interests to disclose. The results of the present study are presented clearly, honestly, and without fabrication do not constitute endorsement by ACSM. There was no external funding received for this research.

\section{Running title: $\beta$-alanine enhances sprint-interval training}




\section{ABSTRACT}

Purpose: The present study investigated the effects of $\beta$-alanine supplementation only, and in combination with sprint-interval training (SIT), on training intensity, and energy provision and performance during exhaustive supramaximal-intensity cycling and a 4- and 10-km time trial (TT). Methods: Fourteen trained cyclists $\left(\mathrm{VO}_{2 \max }=4.5 \pm 0.6 \mathrm{~L} \cdot \mathrm{min}^{-1}\right)$ participated in this placebo-controlled, double-blind study. Subjects performed a supramaximal cycling test to exhaustion (equivalent to $120 \% \mathrm{VO}_{2 \max }$ ) and a $4-$ and $10-\mathrm{km}$ TT and $4 \mathrm{x} 1-\mathrm{km}$ sprints at three time points: before and after $28 \mathrm{~d}$ of supplementation loading $\left(6.4 \mathrm{~g} \cdot \mathrm{d}^{-1}\right)$ with $\beta$-alanine $(\mathrm{n}=7)$ or a placebo $(n=7)$, and after a 5-wk supervised, SIT program performed twice weekly (repeated 1$\mathrm{km}$ cycling sprints) whilst maintaining supplementation with $\beta$-alanine $\left(1.2 \mathrm{~g} \cdot \mathrm{d}^{-1}\right)$ or a placebo. Results: Following the loading period, sprint 3 and 4 of the $4 \times 1-k m$ sprint intervals were improved with $\beta$-alanine supplementation $(4.5 \pm 3.4 \%$ and $7.0 \pm 4.0 \% ; P<0.05$, respectively). After $5 \mathrm{wk}$ of SIT, training intensity increased in both groups but the change was greater with $\beta$ alanine supplementation $(9.9 \pm 5.0 \%$ vs. $4.9 \pm 5.0 ; P=0.04)$. $\beta$-alanine supplementation also improved supramaximal cycling time to exhaustion to a greater extent than placebo $(14.9 \pm 9.2 \%$ vs. $9.0 \pm 6.9 \% ; P=0.04$ ), while 4 - and $10-\mathrm{km}$ TT performance improved to a similar magnitude in both groups. Following SIT, $\beta$-alanine also increased anaerobic capacity $(5.5 \pm 4.2 \% ; P=$ 0.04), while $\mathrm{VO}_{2 \text { peak }}$ increased similarly in each group $(3.1 \pm 2.9 \%$ vs. $3.5 \pm 2.9 \% ; P<0.05)$.

Conclusion: These findings indicate that $\beta$-alanine supplementation enhances training intensity during SIT and provides additional benefits to exhaustive supramaximal cycling compared to SIT alone. Keywords: SPRINT TRAINING; CARNOSINE; TIME TRIAL; TRAINING AID 


\section{INTRODUCTION}

Carnosine is an intramuscular dipeptide which is thought to function as a proton buffer (2) and/or as a calcium regulator (14). $\beta$-alanine supplementation is the most efficacious nutritional strategy known to augment concentrations of muscle carnosine $(6,36)$. Indeed, there is a growing body of evidence that suggests chronic $\beta$-alanine supplementation provides an ergogenic benefit to trained athletes performing short-duration time trials (TT) (7). More specifically, data from our laboratory (5) suggests that $\beta$-alanine supplementation enhances 4-km cycling TT performance ( $\sim 6 \mathrm{~min}$ ), while others have demonstrated worthwhile improvements in 2000-m rowing TT ( $\sim 7$ $\min )$ (20) and 100- ( 1 $\mathrm{min})$ and 200-m swimming TT ( 2 $\mathrm{min})(13)$.

In addition to dietary supplements (such as $\beta$-alanine), various forms of sprint-interval training (SIT) have been demonstrated to improve short-duration TT performance (i.e. $<10 \mathrm{~min})(18,31$, 38). Although there is no universal definition, a SIT session can be defined as repeated maximal efforts of short-duration (i.e. $<2 \mathrm{~min}$ ) and extreme-intensity or sprint-type (i.e. all-out) exercise interspersed with recovery periods performed at a low-intensity, or with complete rest. Improvement in TT performance in well-trained athletes following the implementation of a SIT program has been attributed to the augmentation of both aerobic and anaerobic metabolic adaptations $(11,38)$, as well as the ability to better manage ionic perturbations in the muscle and blood during exercise (22). Given that $\beta$-alanine supplementation has been shown to increase the amount of work completed during intense, intermittent sprint exercise (39), an interesting question is raised: Does $\beta$-alanine supplementation provide additional benefits to the effect of SIT by allowing athletes to train at a higher interval training intensity and achieve a greater degree of training adaptation and, therefore, performance? 
Recent research has failed to demonstrate a concomitant additive enhancement in physiological or performance measures when $\beta$-alanine is combined with a training intervention $(10,17,24$, 33). This finding may be related in part, to the recruitment of untrained participants in these studies $(10,17,24,33)$ as it is possible that the potent training stimulus may have overwhelmed the potential to detect any influence of $\beta$-alanine supplementation on training intensity and exercise performance. Furthermore, very few studies $(24,33)$ have included a supplementation loading period to elevate carnosine levels prior to the implementation of the training intervention. In addition, these studies $(10,17)$ did not require their subjects to perform their exercise training protocol prior to the supplementation loading period to establish whether $\beta$ alanine supplementation could provide an ergogenic benefit to the chosen training protocol. Thus, the efficacy of $\beta$-alanine supplementation as a training aid remains unclear. Therefore, the aim of the present study was to investigate the effects of $\beta$-alanine supplementation only, and in combination with SIT, on training intensity, and energy provision and performance during exhaustive supramaximal cycling and 4 x 1-, 4- and 10-km TT performance.

\section{METHODOLOGY}

\section{Participants}

Fourteen endurance-trained male cyclists (mean \pm SD: age $=25.4 \pm 7.2 \mathrm{yr}$, mass $=71.1 \pm 7.1 \mathrm{~kg}$, $\mathrm{VO}_{2 \max }=4.5 \pm 0.6 \mathrm{~L} \cdot \mathrm{min}^{-1}$ ) were recruited for the current study. Before preliminary testing, all cyclists completed a training questionnaire to estimate the weekly training frequency, the weekly training hours, and the average weekly frequency of interval training during the preceding

month. During the month prior to the investigation, they were cycling $200-550 \mathrm{~km} \cdot \mathrm{wk}^{-1}, 7-16$ $\mathrm{h} \cdot \mathrm{wk}^{-1}$ and performing $\sim 1.5$ interval sessions per week. All cyclists were informed verbally and 
in writing as to the requirements of the study and any possible adverse effects that could be experienced, and all gave their written informed consent. Cyclists had not taken any dietary supplements in the 3 mo before the study with the exception of four cyclists who were consuming a multi-vitamin supplement and two cyclists who were consuming a fish-oil supplement. All cyclists consumed carbohydrate and protein beverages during and after training rides and all cyclists were naive to chronic $\beta$-alanine supplementation before the commencement of the study. The study was conducted in the Griffith University Sport Science laboratory and was approved by the Griffith University Human Research Ethics Committee.

\section{Experimental design}

The current study was a double-blind placebo-controlled trial. Subjects were matched for average weekly training volume $\left(\mathrm{km} \cdot \mathrm{wk}^{-1}\right)$ in the month preceding the investigation and divided in to a $\beta$-alanine (BA; $\mathrm{n}=7$ ) or placebo group (PL; $\mathrm{n}=7$ ). On the initial visit to the laboratory, subjects performed a long-graded exercise test for the determination of $\mathrm{VO}_{2 \max }$ and peak aerobic power output. Subjects completed a familiarization of each performance test (see below) on separate days following the completion of the long-graded exercise test. Following the completion of preliminary testing, subjects performed a supramaximal cycling test to exhaustion (power output equal to $120 \%$ of the power output achieved at $\mathrm{VO}_{2 \max }$ ), a 4 - and $10-\mathrm{km}$ TT and 4 x 1-km sprints on separate days ( $>48 \mathrm{hrs}$ ) at three time points: before (pre-loading) and after (post-loading) $28 \mathrm{~d}$ of supplementation loading $\left(6.4 \mathrm{~g} \cdot \mathrm{day}^{-1}\right)$ with $\beta$-alanine or a placebo; and after a 5-wk supervised SIT program (post-SIT) performed twice weekly whilst maintaining supplementation $\left(1.2 \mathrm{~g} \cdot \mathrm{day}^{-1}\right)$. Subjects recorded all types of training and the duration and 
intensity of each training session throughout the entirety of the study using the session-rating of perceived exertion (RPE) method (16).

\section{Details of exercise performance tests}

Before reporting to the laboratory for each performance test, each subject was asked to abstain from caffeine and alcohol and avoid strenuous exercise for the $24 \mathrm{~h}$ preceding each performance test. Each subject was also required to record a 24-h diet diary leading up to the first performance test, which was then replicated in the $24 \mathrm{~h}$ preceding the performance tests. All testing was conducted on an electromagnetically-braked cycle ergometer (Velotron Racermate, Seattle, WA) Prior to each test, factory calibration was verified using the Accuwatt "run down" verification program (RacerMate Inc.) accompanying the ergometer software. The ergometer was adjusted for comfort for each subject; this included fitting their own pedals and saddle. These adjustments were replicated for all subsequent trials. Subjects commenced a standardized 20-min warm-up before each performance trial that comprised 5 min of cycling at $150 \mathrm{~W}, 8$ min of cycling at a power output equivalent to $60 \%$ of the power output achieved at $\mathrm{VO}_{2 \max }, 2$ min of cycling at a self-selected power output, 2 min of cycling to include three, 6-s maximal sprints and finished with $3 \mathrm{~min}$ of cycling at a self-selected power output. Subjects were then instructed to sit passively for a period of $8 \mathrm{~min}$ before starting the performance test.

\section{Long-graded exercise test}

Subjects completed the long-graded exercise test which required the subjects to begin cycling at $100 \mathrm{~W}$ with the work rate increasing by $50 \mathrm{~W}$ every $5 \mathrm{~min}$ until volitional exhaustion or if their pedalling frequency dropped below $60 \mathrm{rev} \cdot \mathrm{min}^{-1}$. Gas exchange variables were measured and 
recorded every $30 \mathrm{~s}$ (Parvomedics Trueone 2400, Utah, USA). Maximal aerobic power output was calculated from the last completed power output stage, plus the fraction of time spent in the final non-completed work rate multiplied by the work rate increment.

\section{Supramaximal cycling tests}

Subjects commenced the standardized warm-up period and were then given $10 \mathrm{~s}$ of unloaded cycling to allow subject to reach a pedalling cadence of approximately $110 \mathrm{rev} \cdot \mathrm{min}^{-1}$ before the predetermined power output equal to $120 \%$ of the power output achieved at $\mathrm{VO}_{2 \max }\left(\mathrm{L} \cdot \mathrm{min}^{-1}\right)$ was applied. To determine the time to exhaustion (TTE), the test was terminated when each cyclist could not maintain a pedalling cadence of above $60 \mathrm{rev} \cdot \mathrm{min}^{-1}$ for a 3 -s period. The coefficient of variation (CV) for TTE (expressed as a CV\% between the familiarization and preloading supramaximal cycling test) was $6.4 \%$. Gas exchange variables were measured and recorded (Parvomedics Trueone 2400, Utah, USA). A blood sample was taken from the earlobe

immediately post-exercise for determination of blood $\left[\mathrm{La}^{\circ}\right]$ using the Lactate Pro ${ }^{\mathrm{TM}}$ (Arkray KDK, Japan).

\section{4- and 10-km cycling TT}

A flat course profile was created using the Velotron Coaching Software (RacerMate Inc., Seattle, USA) for each distance. Subjects commenced the standardized warm-up before beginning each TT. During the TT, subjects were able to see the distance remaining and the gear ratio. The gear ratio $(53 \times 17)$ was the same at the start of each time trial, but subjects were permitted to adjust the gear ratio throughout the trial to reflect their preferred cadence. Power output (Velotron Coaching software, Racermate, USA) was recorded at a frequency of $30 \mathrm{~Hz}$ and heart rate was 
recorded throughout exercise using short-range telemetry (Polar T61, Kempele, Finland), interfaced with the Velotron software. The CVs for performance time for the 4- and 10-km TT were 1.1 and $1.2 \%$. The CVs for mean power output for the 4 - and $10-\mathrm{km}$ TT were 2.2 and $2.3 \%$.

\section{$4 \times 1-k m$ cycling sprints}

Subjects performed $4 \times 1-\mathrm{km}$ maximal cycling sprints with each sprint being separated by 4 min of active recovery ( $\sim 50 \mathrm{~W})$ on an air-braked cycle ergometer (Wattbike Pro, Nottingham, UK). Subjects completed the standardized warm-up prior to the $4 \times 1-\mathrm{km}$ maximal cycling sprints and were encouraged to perform each 1-km maximal sprint in the fastest time possible. Performance time and mean power output were recorded for each $1-\mathrm{km}$ cycling sprint. The CV for performance time $(0.6 \%)$ and mean power output for the $1-\mathrm{km}$ TT $(1.2 \%)$ in trained cyclists has previously been determined to be acceptable in our laboratory (4). Heart rate was recorded throughout exercise (Polar T61, Kempele, Finland) and blood lactate concentration was determined after each 1-km cycling sprint using the Lactate Pro ${ }^{\mathrm{TM}}$ (Arkray KDK, Japan).

\section{Supplementation loading and maintenance protocols}

During the loading period, subjects were supplemented with $6.4 \mathrm{~g} \cdot \mathrm{day}^{-1}$ of $\beta$-alanine (Carnosyn ${ }^{\circledR}$ slow-release, Collegiate Sport Nutrition, San Marcos, California, USA) or a placebo (dextrose monohydrate) ingested in four equal daily doses with for $28 \mathrm{~d}$. Supplements were provided as 400-mg gel capsules, and subjects consumed doses at each of the three main meals and before going to sleep. During the SIT period, subjects decreased their supplement intake to $1.2 \mathrm{~g} \cdot \mathrm{day}^{-1}$ and continued this dose until each subject had completed their last performance test in the post- 
SIT period. Recent research has suggested that a maintenance dose of $\sim 1.2 \mathrm{~g} \cdot$ day $^{-1}$ of $\beta$-alanine is considered appropriate to maintain already elevated muscle carnosine content (35).

\section{Sprint-interval training program}

Following the performance tests conducted after the loading period (post-loading), subjects performed supervised training sessions $2 \mathrm{~d} \cdot \mathrm{wk}^{-1}$ for a total of $5 \mathrm{wk}$ in addition to each subjects normal training regimen. Subjects continued to record all types of training and the duration and intensity of each training session during the SIT period using the session RPE method (16). Each SIT session was comprised of repeated 1-km maximal cycling sprints with each sprint being separated by 4 min of active recovery $(\sim 50 \mathrm{~W})$ performed on an air-braked cycle ergometer (Wattbike Pro, Nottingham, UK). Training progression was implemented by increasing the number of 1-km maximal sprints from four repetitions during session 1-5, to five repetitions during session 6 and 7, and finally to six repetitions during sessions 8 and 9. Subjects completed four repetitions in session 10 to allow for a matched-repetition analysis of training performance for the $4 \times 1-\mathrm{km}$ cycling sprint protocol conducted prior to supplementation (pre-loading) and the first (post-loading) and last (post-SIT) sprint interval training session. Subjects were encouraged to perform each 1-km maximal sprint in the fastest time possible. Performance time, mean and peak power output were recorded for each 1-km cycling sprint for each training session. Prior to each training sessions subjects completed the standardized warm-up. In an attempt to standardize the nutrient availability for each subject prior to each training session, subjects were encouraged to consume a high carbohydrate meal $\left(2-4 \mathrm{~g} \cdot \mathrm{kg}^{-1}\right)$ prior to each training session in an attempt to maximise training performance (9). 


\section{Data analyses}

Anaerobic capacity was determined from the supramaximal cycling tests and was calculated from the power output, $\mathrm{VO}_{2}, \mathrm{RER}$ and gross efficiency by the methods used by Serresse et al. (32) and adapted by Foster et al. (15). Gross efficiency was calculated from the average $\mathrm{VO}_{2}$ and RER from the final 2 min of the warm-up stage that was completed at the power output equivalent to $60 \%$ of the power output achieved at $\mathrm{VO}_{2 \max }$. Power output and cardiorespiratory data were averaged and time-aligned to the start of each test to facilitate between-trial analyses. Subsequently, the average power output and the average $\mathrm{VO}_{2}$ during each interval were calculated and the aerobic contribution to power output was calculated by multiplying metabolic work by gross efficiency. We assumed that RER $>1.0$ were attributable to non-metabolic $\mathrm{CO}_{2}$ production attributable to the buffering action by bicarbonate. Accordingly, RER values in excess of 1.0 were treated as if they equalled 1.0 in the calculation of metabolic work. The anaerobic contribution to power output was calculated by subtracting the aerobic contribution to power output from the total power output. Summation of total anaerobic power output over time provided a measure of anaerobic capacity. We also calculated the post-loading and post-SIT anaerobic capacity at isotime relative to the pre-loading TTE (i.e. work-matched comparison). $\mathrm{VO}_{2 \text { peak }}$ was defined as the highest 30 -s average during each supramaximal cycling test.

\section{Statistical analysis}

The change score in performance and physiological variables for $\beta$-alanine and placebo after the supplementation loading period and again after the training period were compared using a mixed design $2 \times 3$ (group $\times$ testing period) ANOVA with repeated measures for time. Post hoc analyses were performed where appropriate by using pairwise comparisons with Bonferroni 
adjustments. Results are reported as mean \pm standard deviation unless stated otherwise and statistical significance was accepted at the $P<0.05$ level. Where practical, precision of estimates of outcome statistics are reported as $95 \% \mathrm{CL}$ of the difference between conditions, and as probabilities that the true effect is beneficial, trivial, and/or harmful which were determined from a Microsoft Excel spreadsheet designed for sport science research (3). When clear interpretation was able to be made, a qualitative descriptor was assigned to the following quantitative chances of benefit: $0-25 \%$; unclear; $25-75 \%$, benefit possible; $75-95 \%$, benefit likely; $95-99 \%$, benefit very likely; $>99 \%$, benefit almost certain.

\section{RESULTS}

$4 \times 1-k m$ sprints and sprint-interval training

At post-loading, BA significantly improved performance time $(-1.6 \pm 1.0 \%$ and $-2.1 \pm 2.0 \% ; P<$ 0.05 , respectively) and mean power output $(+4.5 \pm 3.4 \%$ and $+7.0 \pm 4.0 \% ; P<0.05$, respectively) in sprint 3 and 4 of the $4 \times 1-\mathrm{km}$ cycling sprints, whilst there was no change in PL (Table 1). Figure 1 shows the transient improvement in training performance times for each 1$\mathrm{km}$ maximal cycling sprint relative to the first training session throughout the entire training period. During training session 6 and 7, BA achieved a significantly greater improvement in performance time for sprint $2(P<0.05)$ compared to PL. During training session 5 through to 10, BA achieved a significantly greater improvement in performance time for sprint 3 and $4(P<$ 0.05) compared to PL. Furthermore, the improvement in mean power output achieved during the pooled 1-km cycling sprints increased from the first to the last training session to a greater extent in BA compared to PL $(\mathrm{BA}:+9.9 \pm 5.0 \%$; PL: $+4.9 \pm 5.0 ; P=0.044)$. During the final training session, blood lactate concentration was significantly higher following sprint 3 (BA: $16.1 \pm 1.9$ 
$\left.\mathrm{mmol} \cdot \mathrm{L}^{-1} ; \mathrm{PL}: 15.0 \pm 1.7 \mathrm{mmol} \cdot \mathrm{L}^{-1} ; P=0.050\right)$ and sprint $4\left(\mathrm{BA}: 16.9 \pm 1.9 \mathrm{mmol} \cdot \mathrm{L}^{-1}\right.$; PL: 15.3 $\left.\pm 1.9 \mathrm{mmol} \cdot \mathrm{L}^{-1} ; P=0.048\right)$ in BA compared to PL.

\section{Supramaximal cycling test}

At post-loading, BA had a significant increase in TTE $(+8.9 \pm 8.4 \% ; P=0.017$; Figure 2$)$ with a corresponding increase in anaerobic capacity $(+4.0 \pm 3.0 \% ; P=0.018)$ and post-exercise blood lactate concentration $(+8.1 \pm 8.5 \% ; P=0.038)$, whilst there was no change in PL (Table 2). At post-SIT, both groups had improvements in TTE $(\mathrm{BA}:+14.9 \pm 9.2 \%$; PL: $+9.0 \pm 6.9 \%$; $P<$ 0.05). However, there was a greater improvement in BA compared to PL (a difference of $+15.0 \pm$ $13.5 \mathrm{~s}$ between the $\Delta \mathrm{BA}-\Delta \mathrm{PL}, P=0.040$; table 3 ). In addition, $\mathrm{BA}$ had a significant improvement in anaerobic capacity $(+5.5 \pm 4.3 \% ; P=0.040)$, whilst there was no change in PL. We also examined the post-loading and post-SIT anaerobic capacity measured at the time equal to the TTE of the pre-loading supramaximal cycling test (i.e. work-matched comparison). There was no significant difference in the relative post-loading anaerobic capacity matched to the preloading TTE in BA or PL (Figure 3). Similarly, there was no difference in the relative post-SIT anaerobic capacity matched to the pre-loading TTE in the BA or PL. $\mathrm{VO}_{2 \text { peak }}$ was significantly enhanced to a similar magnitude at post-SIT (BA: $+3.1 \pm 2.9 \%$; PL: $+3.5 \pm 2.9 \% ; P<0.05$ ).

\section{4-km TT}

At post-loading, BA significantly improved 4-km TT performance time $(1.7 \pm 1.7 \% ; P=0.040)$ and mean power output $(+4.1 \pm 4.0 \% ; P=0.030)$, while there was no change observed for PL (Figure 2). At post-SIT, performance time (BA: $-4.0 \pm 1.5 \%$; PL: $-2.9 \pm 1.5 \%$ ) and mean power output (BA: $+10.6 \pm 3.9 \%$; PL: $+8.6 \pm 5.1 \%$ ) improved in both groups (main effect for time, $P<$ 
0.001). Despite there being a non-significant group $\mathrm{x}$ time interaction at post-SIT, there was a possibly likely (51\% likelihood of a beneficial effect) additive benefit for 4-km TT performance time in BA compared with PL (a difference of $-3.6 \pm 6.0 \mathrm{~s}$ between the $\Delta \mathrm{BA}-\Delta \mathrm{PL}$ ) (Table 3).

\section{$10-\mathrm{km} T T$}

There was no difference in 10-km TT performance time or mean power output for either group at post-loading (Figure 2). At post-SIT, there was a significant improvement in 10-km TT performance time (BA: $-1.4 \pm 1.3 \%$; PL: $-1.5 \pm 1.4 \% ; P<0.05$ ) and mean power output (BA: $+3.9 \pm 3.7 \%$; PL: $+4.3 \pm 4.1 \% ; P<0.05)$ in both groups. However, the magnitude of the increase in performance was not different between groups.

Blinding efficacy and supplementation compliance

Compliance with the supplementation protocol was verbally confirmed from all subjects. All subjects were free of paraesthesia symptoms during the supplementation period. We also asked all subjects to guess which group ( $\beta$-alanine or placebo) they were in during the debriefing session that we held with each subject once all subjects had completed their study requirements. All subjects were hesitant to guess which group they were in but only two out of the seven subjects in each group successfully predicted which group they were in.

\section{DISCUSSION}

The present study demonstrates that $\beta$-alanine supplementation increases training intensity during extreme-intensity bouts of cycling sprints (i.e. SIT). Importantly, the increase in training intensity provided additional benefits to cyclists performing exhaustive supramaximal cycling 
compared to SIT alone. We also showed that these additive performance improvements were associated with an enhanced anaerobic capacity during supramaximal cycling.

Considering we have previously reported performance improvements during supramaximalintensity cycling and 4-km cycling TT (5) following $\beta$-alanine supplementation, the focus of the current study was to investigate whether $\beta$-alanine supplementation could provide added benefits to the effect of SIT on cycling performance by further inducing training adaptations $(10,17)$. The premise that may support the use of $\beta$-alanine as a training aid relies on the ergogenic potential of $\beta$-alanine to enhance performance during the employed training protocol (i.e. increase training intensity). A novel aspect of the current study is that we required our participants to perform the SIT protocol during the pre-loading testing period in order to determine whether the training protocol (repeated 1-km maximal efforts) could be improved following supplementation with $\beta$ alanine. Indeed, BA improved mean power output in sprint 3 and 4 of the $4 \times 1-\mathrm{km}$ sprints $(+4.5$ $\pm 3.4 \%$ and $+7.0 \pm 4.0 \% ; P<0.05$, respectively) with no change in PL. We adopted an identical training protocol for a 5-wk training period and as hypothesized, commencing SIT following supplementation loading with $\beta$-alanine supplementation, and presumably elevated levels of muscle carnosine (36), resulted in higher power outputs during SIT sessions which was most pronounced in the latter 1-km cycling sprints. The improvement in mean power output achieved during the 1-km cycling sprints increased from the first to the last training session to a greater extent in BA compared to PL (BA: $+9.9 \pm 5.0 \%$; PL: $+4.9 \pm 5.0 ; P=0.044)$.

In the present study, SIT alone induced improvements in supramaximal exercise tolerance and 4and 10-km TT performance. Several previous studies have examined muscle metabolic and/or 
performance adaptations to SIT in well-trained endurance athletes $(11,22,31,38)$. For example, Rønnestad et al., (31) reported improvements in $\mathrm{VO}_{2 \max }$ and various performance parameters including mean power output during a 30-s all-out, 5-min all-out, and 40-min all-out cycling tests following a 10-wk SIT program in competitive male cyclists. Furthermore, Iaia et al., (22) reported an attenuation in plasma $\mathrm{K}^{+}$concentration following a 4-wk SIT program in endurance trained runners concomitant with improvements in exhaustive supramaximal exercise tolerance. One of the main findings from the present study was that supramaximal cycling TTE increased to a greater extent following SIT in cyclists who supplemented with $\beta$-alanine, compared with a placebo. Furthermore, there was a non-statistical trend for a greater improvement in 4-km TT performance time following SIT in BA $(4.0 \pm 1.5 \%)$, compared to PL $(2.9 \pm 1.5 \%)$, whilst 10-km TT performance time improved modestly and to a similar extent in both groups (BA: $1.3 \pm 1.5 \%$; PL: $1.4 \pm 1.4 ; P=0.46)$. The additional $1.1 \%$ improvement in $4-\mathrm{km}$ TT performance time in BA did not reach statistical significance. Indeed, the small sample size in the current study $(\mathrm{n}=14)$ may have been insufficient to provide adequate statistical power to detect a significant performance improvement with traditional null hypothesis testing. However, given that performances of elite athletes are separated by very small margins that would be difficult to statistically detect (21), the apparent trend towards a greater improvement in performance in BA at post-training may be important in practical terms. Our finding of an increased training intensity and superior improvement in supramaximal-intensity exercise to exhaustion in response to undertaking SIT following $\beta$-alanine supplementation is in contrast to two recent studies that have also investigated the effects of $\beta$-alanine supplementation as a training aid for interval training $(10,17)$. However, limitations with the training and testing protocols and the training 
status of the subjects have made it difficult to extrapolate the results of these investigations to the current study.

Gross et al. (17) required participants to perform 90 -s of severe intensity (110\% peak power output) cycling both before and after $\beta$-alanine supplementation ( $3.2 \mathrm{~g} \cdot \mathrm{d}-1$ for $38 \mathrm{~d}$ ) and after an 11-d high-intensity interval training (HIT) block (9 sessions; 4 x 4 min @ 90-95\% maximum HR), which followed supplementation. Importantly, supplementation was halted prior to the training intervention which resulted in an $8 \%$ reduction in the augmented carnosine content throughout the training block which may have reduced the efficacy of the supplementation loading phase. Furthermore, the subjects in the study were previously untrained individuals who performed an exercise test that was fixed in duration and intensity which is not representative of a true competitive performance protocol. In addition, the 11-day HIT block may not have allowed for adequate recovery time between training sessions to promote recovery and facilitate performance adaptations, particularly in the untrained subjects recruited in the study. The importance of rest days between interval training sessions was emphasized in a recent study (28) that showed peak and mean power output elicited during a Wingate test were unchanged after fourteen consecutive days of sprint training; however, when subjects performed the same number of training sessions over 6 wk (i.e., with 1-2 days of rest between training sessions), power output improved significantly.

Cochran et al. (10) studied the effects of $\beta$-alanine supplementation on training performance during a 6-wk SIT intervention (three weekly sessions consisting of 4-6 Wingate tests) and cycling performance tests (250-kJ time-trial and a repeated sprint test) performed pre- and post- 
training. The authors reported that the total work performed during each training session was similar between the placebo and $\beta$-alanine group, as well as markers of mitochondrial content, $\mathrm{VO}_{2 \text { peak }}$, repeated-sprint capacity, and 250-kJ time trial performance. Several major differences in the training protocols between the present study and that of Cochran et al. (10) are likely to be responsible for the contrasting effects on performance. First, we chose to prescribe distancebased intervals (1-km) compared to duration-based intervals (30-s) (17). Recent research (1) has suggested that individuals undertake duration-based cycling trials with a more conservative pacing strategy compared to distance-based cycling trials which may be a confounding factor (29) and mask any ergogenic potential that supplementation with $\beta$-alanine can provide. Second, we required our participants to perform our SIT protocol prior to supplementation to ensure that $\beta$-alanine supplementation had the potential to increase training intensity, which was an omission from the study of Cochran et al. (10). We were able to demonstrate a significant improvement in mean power output and performance time in sprint 3 and 4 of the $4 \times 1-\mathrm{km}$ cycling sprints following $28 \mathrm{~d}$ of $\beta$-alanine supplementation which ensured that our SIT protocol provided the appropriate opportunity to allow the cyclists to increase their training intensity in response to $\beta$ alanine supplementation. Third, we utilized cyclists who we considered "endurance-trained" $\left(\mathrm{VO}_{2 \max }=4.5 \pm 0.6 \mathrm{~L} \cdot \mathrm{min}^{-1}\right)(12)$ before entering the study, compared with previously healthy but untrained subjects included in the study of Cochran et al. (10). Indeed, the training stimulus may have overwhelmed any potential influence of $\beta$-alanine supplementation on physiological or performance measures in the untrained participants recruited for the study of Cochran et al. (10).

In the present study, the superior improvement in exhaustive supramaximal exercise following SIT in BA was accompanied by an augmentation in anaerobic capacity, while there was no 
change in anaerobic capacity following training in the PL. Indeed, the increase in the absolute anaerobic energy yield in BA may explain the superior improvement in exhaustive supramaximal-intensity cycling compared to PL. Considering BA trained at a higher intensity throughout the SIT program, it may suggest that improvements in anaerobic capacity are intensity-dependant. It has been demonstrated that muscle contents of ATP and $\mathrm{PCr}$ are not changed by SIT (27). However, anaerobic glycolysis is a key metabolic process that accounts for $\sim 70-80 \%$ of anaerobic capacity (26). Thus, augmentation of anaerobic glycolysis is likely to be the main metabolic pathway accounting for the increase in anaerobic capacity. The possible mechanisms that contribute to augmented anaerobic glycolysis during intense exercise after SIT may include an increase in glycolytic flux rate due, in part, to higher phosphofructokinase and/or hexokinase activity $(23,25)$. However, this has not been a universal finding in well-trained athletes following interval training as Weston et al. (40) reported that the activities of hexokinase and phosphofructokinase did not change with HIT. However, it is possible that the intervals used by Weston et al. (40) were performed at too low an intensity ( $85 \%$ of peak aerobic power) to provide an appropriate stimulus that would augment glycolytic enzyme activity. Augmentation of glycolytic enzymes may be expected to not only enhance the volume of anaerobic energy production, but also the rate at which ATP is produced from anaerobic glycolysis. In the present study, we contrasted the post-SIT anaerobic capacity measured at the time equal to the TTE of the pre-loading supramaximal cycling test (i.e. work-matched comparison) which revealed remarkably similar values. This suggests that the SIT program employed in the current study did not alter the rate of anaerobic ATP production during supramaximal cycling. Rather, the anaerobic capacity in BA increased as a result of the prolonged exercise duration following SIT. Therefore, we suggest that the augmented absolute anaerobic capacity is a result of an enhanced 
muscle buffering capacity which has been reported to be an important physiological adaptation in trained individuals from both HIT (40) and SIT (37). A high concentration of $\mathrm{H}^{+}$ions has a known inhibitory effect on glycolytic enzyme activity, including phosophofructokinase (34). Thus, improved skeletal muscle buffering capacity could indirectly contribute to an improved absolute glycolytic ATP yield by prolonging the activity of phosophofructokinase by better managing ionic perturbations during intense exercise. Although further examination of this mechanism is required, augmentation in skeletal muscle buffering capacity is a prospective mechanism that may have enhanced anaerobic capacity in BA following SIT.

In addition to improvements in anaerobic capacity, BA displayed significantly higher values of blood lactate concentration during the final SIT session. One of the mechanisms behind the efficacy of SIT for exercise performance may be related to increased lactate exposure which is suggested to increase mitochondrial biogenesis and the expression of lactate transporters (8) which may improve the ability of the muscle to release lactate and $\mathrm{H}^{+}$during intense contractions and enhance high-intensity endurance performance (30). Indeed, it has been reported that the lactate anion affects muscle-signalling pathways, leading to an increase in cytochrome $c$ oxidase mRNA and protein, and also PGC-1 $\alpha$ expression (19). The augmented anaerobic capacity in BA may have resulted in an acceleration of glycogenolysis, resulting in a mismatch between the rates of pyruvate production and oxidation, which lead to the increase in lactate production. Thus, if lactate is an important signalling molecule, greater increases in muscle lactate production during training in BA may have served to promote even greater trained-induced muscular adaptations compared to PL. 
In summary, the present study has shown that $5 \mathrm{wk}$ of SIT, by subjects who are already well trained, accompanied by chronic $\beta$-alanine supplementation, leads to robust changes in supramaximal cycling performance and anaerobic capacity. $\beta$-alanine supplementation increased training intensity during extreme-intensity bouts of cycling sprints (i.e. SIT) which provided additional benefits to cyclists performing exhaustive supramaximal-intensity cycling compared to SIT alone. The additional improvement in supramaximal-intensity cycling following SIT and $\beta$-alanine supplementation does not seem to be explained by differences in $\mathrm{VO}_{2 \text { peak }}$ but is likely to be associated with the greater improvements in anaerobic capacity. 


\section{Acknowledgments}

The authors have no financial relationships relevant to this article to disclose and no competing interests to disclose. The results of the present study are presented clearly, honestly, and without fabrication do not constitute endorsement by ACSM. There was no external funding received for this research. 


\section{REFERENCES}

1. Abbiss C, R., Thompson K, G. , Lipski M, Meyer T, Skorski S. Pacing differs between time- and distance-based time trials in trained cyclists. Int J Sports Physiol Perf. 2015. EPUB. DOI: http://dx.doi.org/10.1123/ijspp.2015-0613

2. Baguet A, Koppo K, Pottier A, Derave W. $\beta$-Alanine supplementation reduces acidosis but not oxygen uptake response during high-intensity cycling exercise. Eur J Appl Physiol. 2010;108(3):495-503.

3. Batterham AM, Hopkins WG. Making meaningful inferences about magnitudes. Sportscience. 2005;9:6-13.

4. Bellinger PM, Minahan C. Reproducibility of a laboratory based 1-km Wattbike cycling time trial in competitive cyclists. J Sci Cycling. 2014;3(3):23-8.

5. Bellinger PM, Minahan CL. Metabolic consequences of $\beta$-alanine supplementation during exhaustive supramaximal cycling and 4000-m time trial performance Appl Physiol Nutr Metab. 2016. EPUB. DOI: 10.1139/apnm-2016-0095

6. Bex T, Chung W, Baguet A et al. Muscle carnosine loading by $\beta$-alanine supplementation is more pronounced in trained vs. untrained muscles. J Appl Physiol. 2014;116(2):204-9.

7. Blancquaert L, Everaert I, Derave W. $\beta$-alanine supplementation, muscle carnosine and exercise performance. Curr Opin Clin Nutr Metab Care. 2015;18(1):63-70.

8. Brooks GA. Cell-cell and intracellular lactate shuttles. J Physiol. 2009;587(23):5591600.

9. Burke LM, Hawley JA, Wong SHS, Jeukendrup AE. Carbohydrates for training and competition. J Sports Sci. 2011;29(sup1):S17-S27. 
10. Cochran A, Percival M, Thompson S et al. $\beta$-Alanine supplementation does not augment the skeletal muscle adaptive response to six weeks of sprint interval training. Int J Sport Nutr Exerc Metab. 2015;25(6):541-49.

11. Creer A, Ricard M, Conlee R, Hoyt G, Parcell A. Neural, metabolic, and performance adaptations to four weeks of high intensity sprint-interval training in trained cyclists. Int J Sports Med. 2004;25(2):92-8.

12. De Pauw K, Roelands B, Cheung SS, De Geus B, Rietjens G, Meeusen R. Guidelines to classify subject groups in sport-science research. Int J Sports Physiol Perform. 2013;8(2):111-22. 13. de Salles Painelli V, Roschel H, de Jesus F et al. The ergogenic effect of $\beta$-alanine combined with sodium bicarbonate on high-intensity swimming performance. Appl Physiol Nutr Metab. 2013;38(5):525-32.

14. Dutka TL, Lamboley CR, McKenna MJ, Murphy RM, Lamb GD. Effects of carnosine on contractile apparatus $\mathrm{Ca}^{2+}$ sensitivity and sarcoplasmic reticulum $\mathrm{Ca}^{2+}$ release in human skeletal muscle fibers. J Appl Physiol. 2012;112(5):728-36.

15. Foster C, De Koning JJ, Hettinga F et al. Pattern of energy expenditure during simulated competition. Med Sci Sports Exerc. 2003;35(5):826-31.

16. Foster C, Florhaug JA, Franklin J et al. A new approach to monitoring exercise training. $J$ Strength Cond Res. 2001;15(1):109-15.

17. Gross $\mathrm{M}$, Boesch $\mathrm{C}$, Bolliger $\mathrm{C}$ et al. Effects of $\beta$-alanine supplementation and interval training on physiological determinants of severe exercise performance. Eur J Appl Physiol. 2014;114(2):221-34.

18. Gunnarsson TP, Bangsbo J. The 10-20-30 training concept improves performance and health profile in moderately trained runners. J Appl Physiol. 2012;113(1):16-24. 
19. Hashimoto T, Hussien R, Oommen S, Gohil K, Brooks GA. Lactate sensitive transcription factor network in L6 cells: activation of MCT1 and mitochondrial biogenesis. FASEB J. 2007;21(10):2602-12.

20. Hobson RM, Harris RC, Martin D et al. Effect of $\beta$-alanine, with and without sodium bicarbonate, on 2000-m rowing performance. Int J Sports Nutr Exerc Metab. 2013;23(5):480-7.

21. Hopkins WG. Measures of reliability in sports medicine and science. Sports Med. 2000;30(1):1-15.

22. Iaia FM, Thomassen M, Kolding $\mathrm{H}$ et al. Reduced volume but increased training intensity elevates muscle $\mathrm{Na}+\mathrm{K}+$ pump $\alpha 1$-subunit and NHE1 expression as well as short-term work capacity in humans. Am J Physiol Regul Integr Comp Physiol. 2008;294(3):966-74.

23. Jacobs I, Esbjörnsson M, Sylven C, Holm I, Jansson E. Sprint training effects on muscle myoglobin, enzymes, fiber types, and blood lactate. Med Sci Sports Exerc. 1987;19(4):368-74.

24. Kendrick I, Harris $\mathrm{R}$, Kim $\mathrm{H}$ et al. The effects of 10 weeks of resistance training combined with $\beta$-alanine supplementation on whole body strength, force production, muscular endurance and body composition. Amino Acids. 2008;34(4):547-54.

25. MacDougall JD, Hicks AL, MacDonald JR, McKelvie RS, Green HJ, Smith KM. Muscle performance and enzymatic adaptations to sprint interval training. $J$ Appl Physiol. $1998 ; 84(6): 2138-42$.

26. Medbo JI, Mohn AC, Tabata I, Bahr R, Vaage O, Sejersted OM. Anaerobic capacity determined by maximal accumulated $\mathrm{O}_{2}$ deficit. J Appl Physiol. 1988;64(1):50-60.

27. Nevill ME, Boobis LH, Brooks S, Williams C. Effect of training on muscle metabolism during treadmill sprinting. J Appl Physiol. 1989;67(6):2376-82. 
28. Parra J, Cadefau JA, Rodas G, AmigÓ N, CussÓ R. The distribution of rest periods affects performance and adaptations of energy metabolism induced by high-intensity training in human muscle. Acta Physiol Scand. 2000;169(2):157-65.

29. Paton C, Hopkins W. Tests of cycling performance. Sports Med. 2001;31(7):489-96.

30. Pilegaard H, Domino K, Noland $\mathrm{T}$ et al. Effect of high-intensity exercise training on lactate $/ \mathrm{H}^{+}$transport capacity in human skeletal muscle. Am J Physiol Endocrinol Metab. 1999;276(2):255-61.

31. Rønnestad B, Hansen J, Vegge G, Tønnessen E, Slettaløkken G. Short intervals induce superior training adaptations compared with long intervals in cyclists-An effort-matched approach. Scand J Med Sci Sports. 2015;25(2):143-51.

32. Serresse O, Lortie G, Bouchard C, Boulay MR. Estimation of the contribution of the various energy systems during maximal work of short duration. Int J Sports Med. 1988;9(6):45660.

33. Smith A, Moon J, Kendall K et al. The effects of $\beta$-alanine supplementation and highintensity interval training on neuromuscular fatigue and muscle function. Eur J Appl Physiol. 2009;105(3):357-63.

34. Spriet L, Soderlund K, Bergstrom M, Hultman E. Skeletal muscle glycogenolysis, glycolysis, and pH during electrical stimulation in men. J Appl Physiol. 1987;62(2):616-21.

35. Stegen S, Bex T, Vervaet C, Vanhee L, Achten E, Derave W. The $\beta$-alanine dose for maintaining moderately elevated muscle carnosine levels. Med Sci Sports Exerc. 2014;46(7): $1426-1432$. 
36. Stellingwerff T, Decombaz J, Harris R, Boesch C. Optimizing human in vivo dosing and delivery of $\beta$-alanine supplements for muscle carnosine synthesis. Amino Acids. 2012;43(1):5765.

37. Stepto NK, Hawley JA, Dennis SC, Hopkins WG. Effects of different interval-training programs on cycling time-trial performance. Med Sci Sports Exerc. 1999;31:736-41.

38. Stevens AW, Olver TT, Lemon PW. Incorporating sprint training with endurance training improves anaerobic capacity and 2,000-m erg performance in trained oarsmen. J Strength Cond Res. 2015;29(1):22-8.

39. Tobias G, Benatti F, Salles Painelli V et al. Additive effects of $\beta$-alanine and sodium bicarbonate on upper-body intermittent performance. Amino Acids. 2013;45(2):309-17.

40. Weston AR, Myburgh KH, Lindsay FH, Dennis SC, Noakes TD, Hawley JA. Skeletal muscle buffering capacity and endurance performance after high-intensity interval training by well-trained cyclists. Eur J Appl Physiol. 1996;75(1):7-13. 


\section{FIGURE CAPTIONS}

Figure 1 - Improvement (\%) in performance time in the first (A), second (B), third (C) and fourth (D) 1-km cycling sprint (4 x 1-km) throughout the 5-wk SIT training period relative to the first training session for the for the $\beta$-alanine group (BA) and placebo group (PL).

*Denotes a significant differences between groups

Figure 2 - Improvement (\%) in time to exhaustion (top panel), 4-km TT performance time (middle panel) and 10-km TT performance time (bottom panel) following 4 wks of supplementation loading (post-loading) and following the 5-wk sprint-interval training program (post-SIT) relative to pre-loading values for the $\beta$-alanine group (BA) and placebo group (PL).

${ }^{\mathrm{a}}$ Denotes significant differences between groups; post-loading vs. post-loading

${ }^{\mathrm{b}}$ Denotes significant differences between groups; post-SIT vs. post-SIT

${ }^{c}$ Denotes significant differences within group; post-SIT vs. post-loading

Figure 3 - Anaerobic capacity measured at iso-time (relative to the time to exhaustion at preloading) and at exhaustion during the supramaximal cycling tests performed prior to supplementation (pre-loading), following 4 wks of supplementation (post-loading) and following the 5-wk SIT program (post-SIT) for the $\beta$-alanine group (BA; left panel) and the placebo group (PL; right panel).

${ }^{a}$ Denotes significant differences within group; post-loading vs. pre-loading

${ }^{b}$ Denotes significant differences within group; post-SIT vs. pre-loading

${ }^{\mathrm{c}}$ Denotes significant differences within group; post-SIT vs. post-loading 


\section{Figure 1}

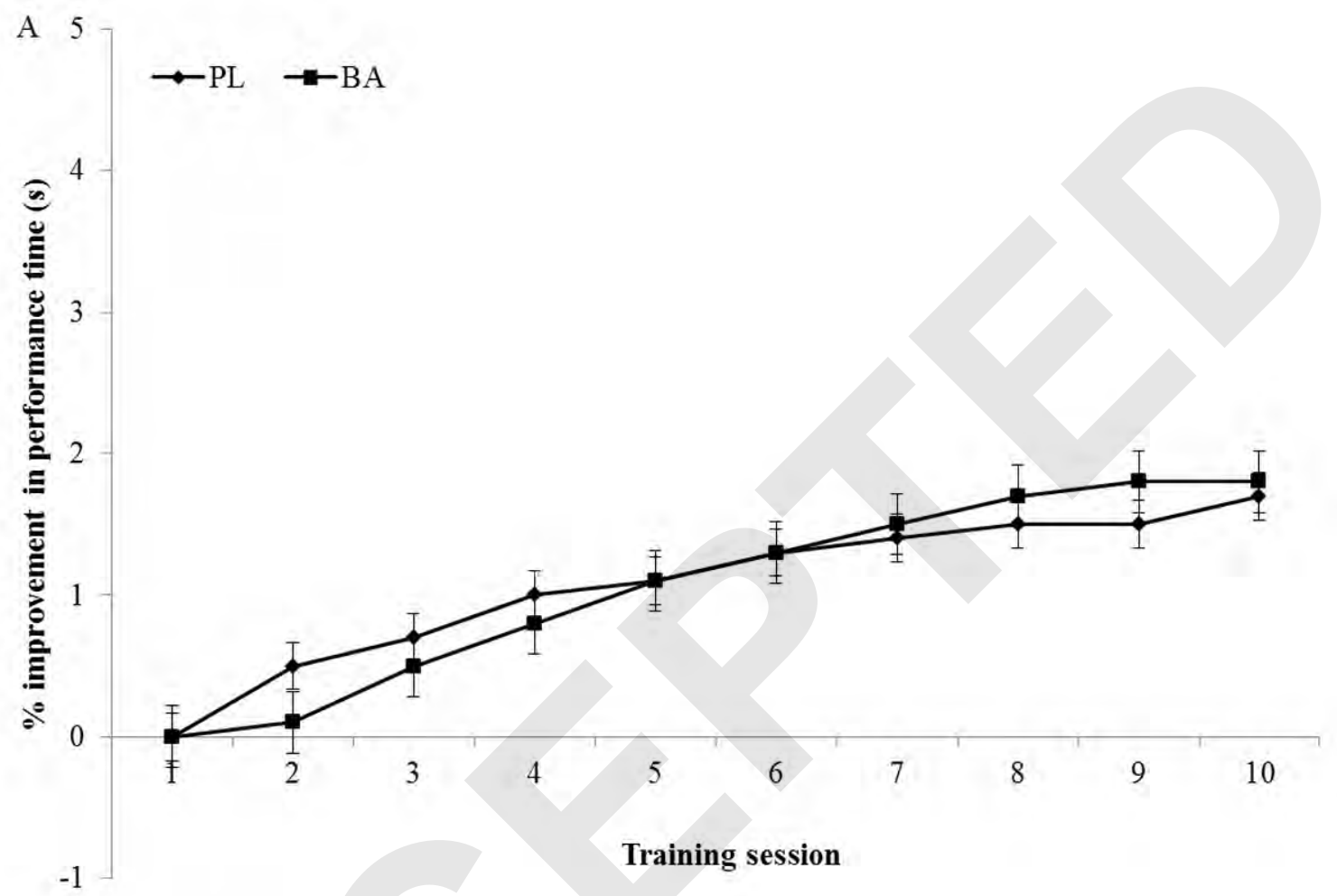




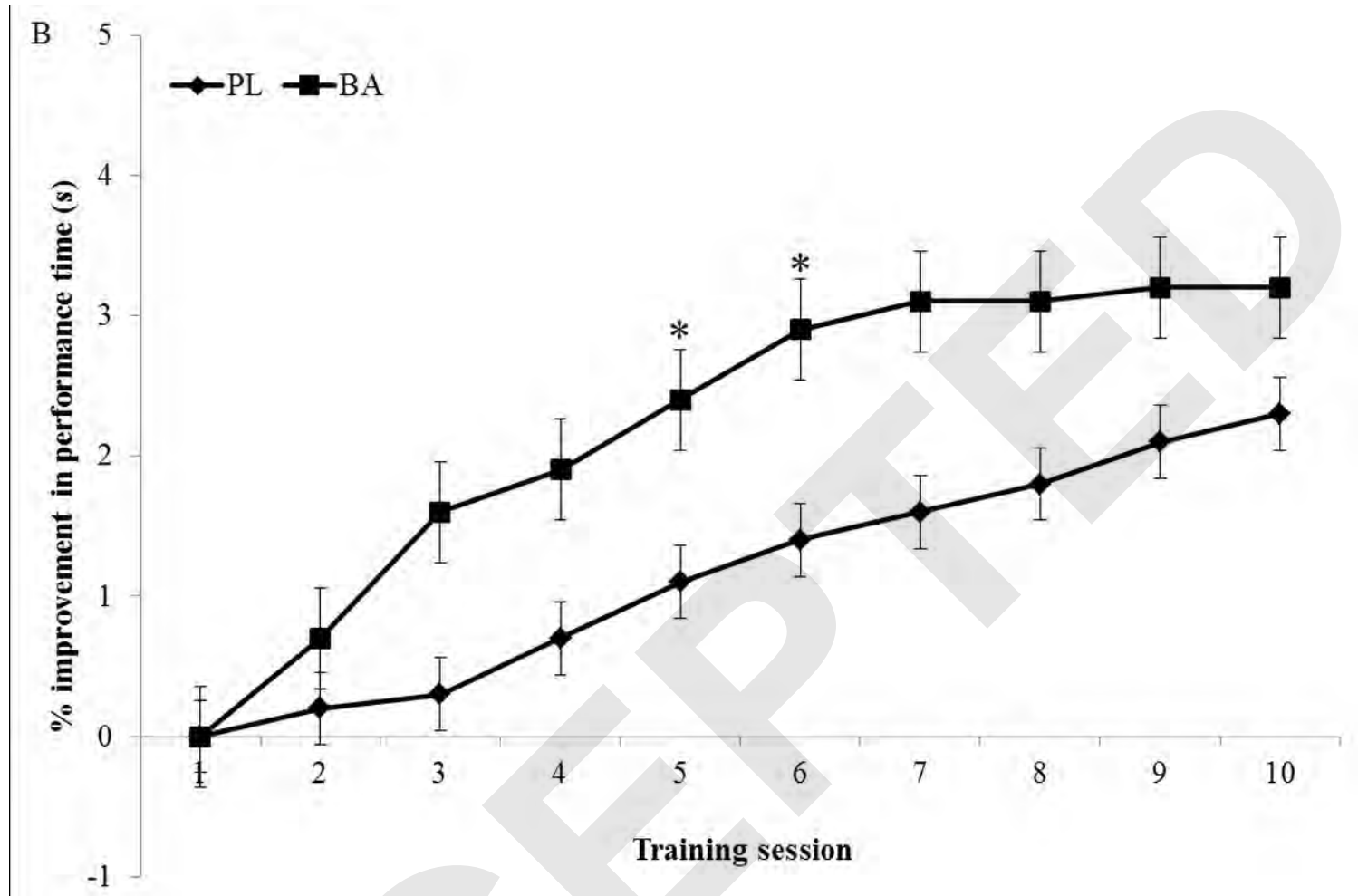




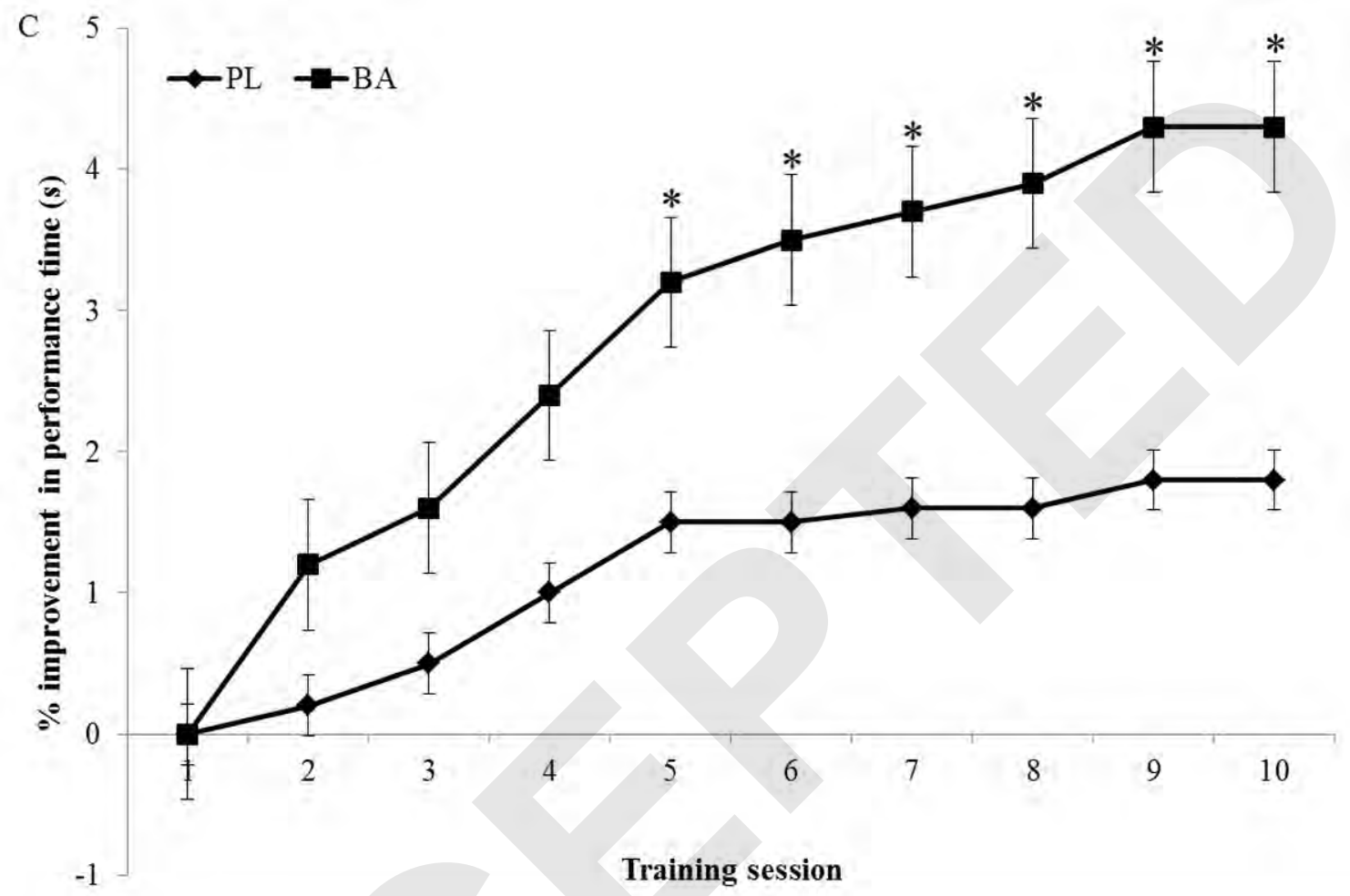




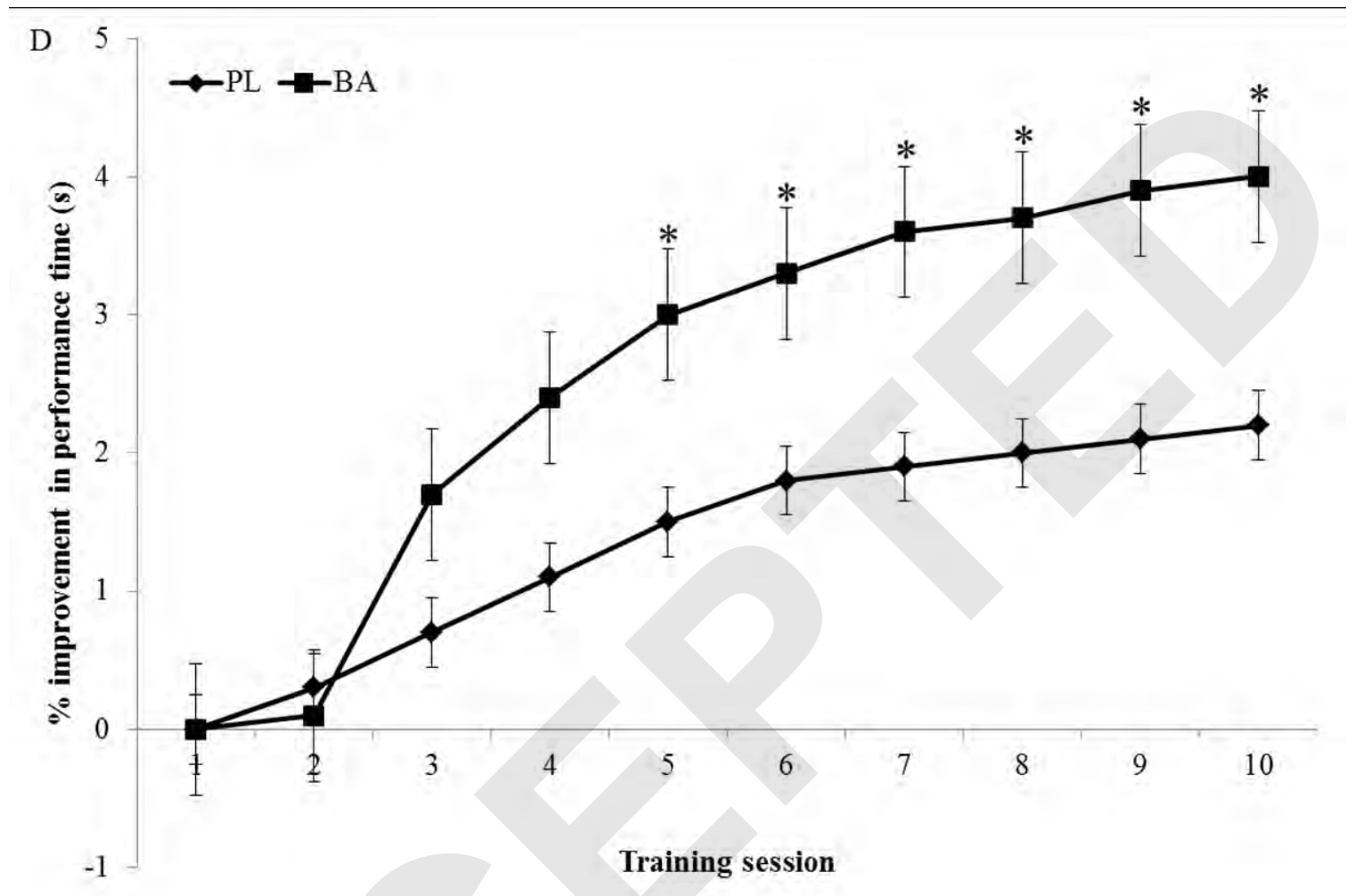

Copyright (C) 2016 by the American College of Sports Medicine. Unauthorized reproduction of this article is prohibited. 
Figure 2
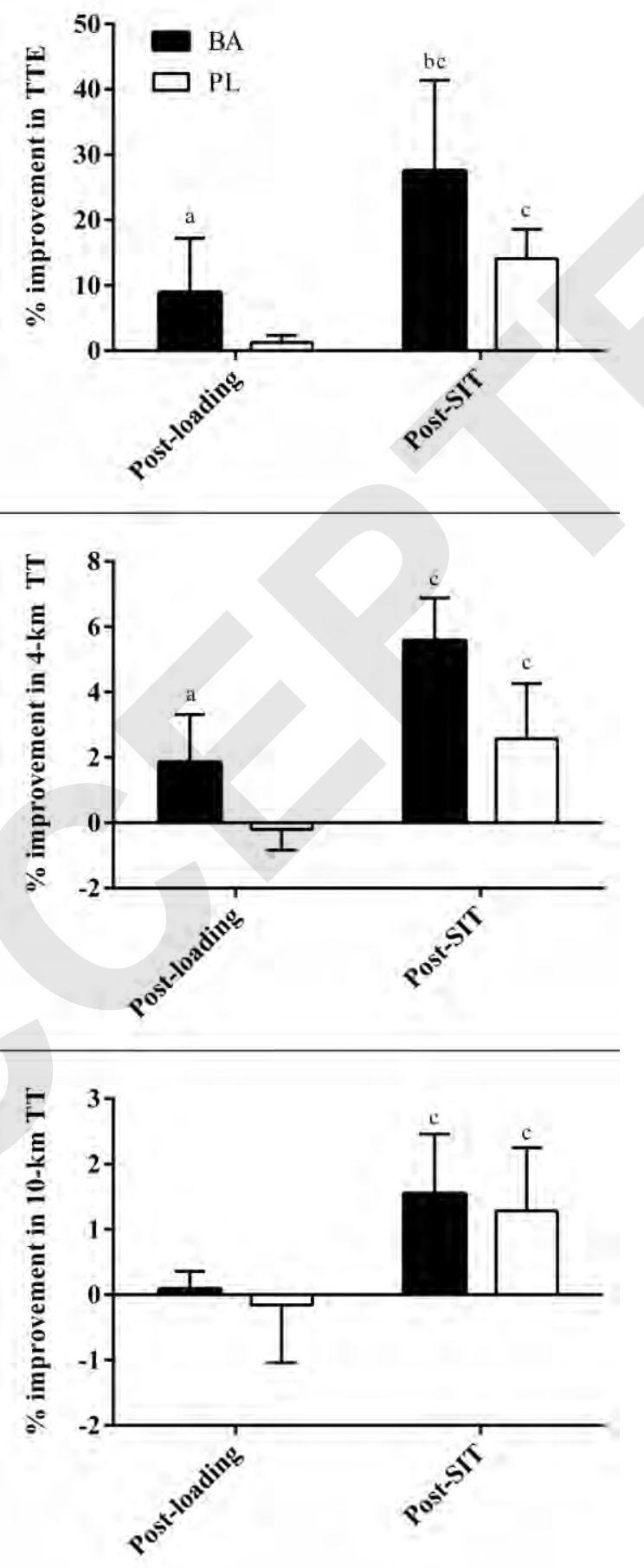


\section{Figure 3}
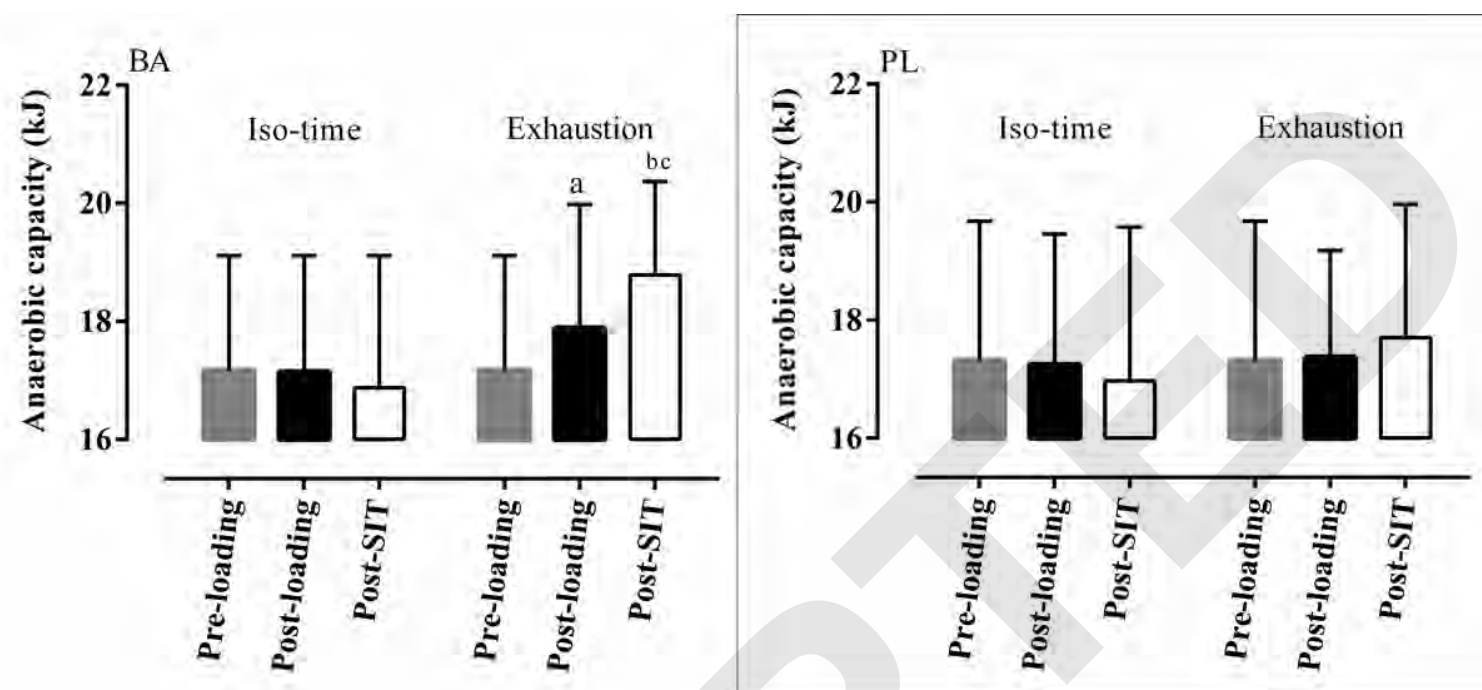
Table 1 - Mean and SEM values for performance time, mean power output and blood lactate concentration for each 1-km cycling sprint conducted prior to supplementation (pre-loading) and the first (post-loading) and last (post-SIT) sprint interval training session for the $\beta$-alanine group (BA) and placebo group (PL).

\begin{tabular}{|c|c|c|c|c|c|c|}
\hline & \multicolumn{3}{|c|}{ PL } & \multicolumn{3}{|c|}{ BA } \\
\hline & Pre-loading & Post-loading & Post-SIT & Pre-loading & Post-loading & Post-SIT \\
\hline \multicolumn{7}{|c|}{ Performance time (s) } \\
\hline 1-km (rep 1) & $70.53 \pm 3.80$ & $70.79 \pm 3.29$ & $69.62 \pm 3.66^{\mathrm{bc}}$ & $70.22 \pm 2.90$ & $70.39 \pm 2.94$ & $69.14 \pm 3.75^{\mathrm{bc}}$ \\
\hline 1-km (rep 2) & $73.94 \pm 2.16$ & $73.93 \pm 2.21$ & $72.26 \pm 2.28^{\mathrm{bc}}$ & $74.14 \pm 2.43$ & $73.60 \pm 3.58$ & $71.48 \pm 3.49^{\mathrm{bc}}$ \\
\hline $1-\mathrm{km}($ rep 3) & $76.97 \pm 3.05$ & $77.12 \pm 3.00$ & $75.71 \pm 2.67^{\mathrm{bc}}$ & $76.74 \pm 2.58$ & $75.50 \pm 3.48^{\mathrm{ad}}$ & $72.22 \pm 3.75^{\text {bce }}$ \\
\hline $1-\mathrm{km}$ (rep 4) & $77.60 \pm 3.20$ & $77.26 \pm 2.86$ & $75.64 \pm 2.63^{b c}$ & $77.11 \pm 2.91$ & $75.49 \pm 3.53^{\mathrm{ad}}$ & $72.45 \pm 3.55^{\text {bce }}$ \\
\hline \multicolumn{7}{|c|}{ Mean power output (W) } \\
\hline 1-km (rep 1) & $505 \pm 57$ & $500 \pm 65$ & $520 \pm 61^{b c}$ & $509 \pm 51$ & $504 \pm 53$ & $527 \pm 65^{\mathrm{bc}}$ \\
\hline $1-\mathrm{km}($ rep 2) & $446 \pm 36$ & $443 \pm 34$ & $477 \pm 38^{\mathrm{bc}}$ & $443 \pm 50$ & $450 \pm 64$ & $490 \pm 58^{\mathrm{bc}}$ \\
\hline $1-\mathrm{km}(\mathrm{rep} 3)$ & $398 \pm 42$ & $396 \pm 43$ & $420 \pm 44^{\mathrm{bc}}$ & $406 \pm 53$ & $423 \pm 63^{\mathrm{ad}}$ & $477 \pm 63^{\text {bce }}$ \\
\hline $1-\mathrm{km}$ (rep 4) & $387 \pm 42$ & $394 \pm 25$ & $421 \pm 27^{\mathrm{bc}}$ & $396 \pm 51$ & $425 \pm 65^{\mathrm{ad}}$ & $473 \pm 65^{\text {bce }}$ \\
\hline \multicolumn{7}{|c|}{ Blood lactate $\left(\mathrm{mmol} \cdot \mathrm{L}^{-1}\right)$} \\
\hline 1-km (rep 1) & $12.6 \pm 3.8$ & $12.7 \pm 3.5$ & $13.4 \pm 3.3$ & $12.3 \pm 1.5$ & $12.5 \pm 1.4$ & $13.1 \pm 1.3$ \\
\hline $1-\mathrm{km}($ rep 2) & $15.0 \pm 1.9$ & $14.6 \pm 1.5$ & $14.9 \pm 2.0$ & $14.9 \pm 2.0$ & $14.9 \pm 1.4$ & $15.8 \pm 2.3^{\mathrm{b}}$ \\
\hline 1-km (rep 3) & $15.2 \pm 1.6$ & $14.8 \pm 1.3$ & $15.0 \pm 1.7$ & $14.7 \pm 1.2$ & $15.0 \pm 1.1$ & $16.1 \pm 1.9^{\text {bce }}$ \\
\hline 1-km (rep 4) & $15.4 \pm 1.8$ & $15.1 \pm 1.9$ & $15.3 \pm 1.9$ & $15.8 \pm 1.6$ & $16.1 \pm 1.8^{\mathrm{d}}$ & $16.9 \pm 1.9^{\text {be }}$ \\
\hline
\end{tabular}

Note. $\mathrm{BA}=\beta$-alanine group; $\mathrm{PL}=$ placebo group; $\mathrm{SIT}=$ sprint-interval training; $\mathrm{SEM}=$ standard error of the mean.

Significant differences within group; ${ }^{\mathrm{a}}$ post-loading vs. pre-loading, ${ }^{\mathrm{b}}$ post-SIT vs. pre-loading, ${ }^{\mathrm{c}}$ post-SIT vs. post-loading.

Significant differences between groups; ${ }^{\mathrm{d}}$ post-loading vs. post-loading, ${ }^{\mathrm{e}}$ post-SIT vs. post-SIT 
Table 2 - Mean and SEM values for time to exhaustion (TTE), anaerobic capacity, $\mathrm{VO}_{2 \text { peak, }}$ and blood lactate concentration measured during the supramaximal cycling tests to exhaustion conducted prior to supplementation (pre-loading), following 4 weeks of supplementation loading (post-loading) and following the 5-wk sprint interval training program (post-SIT) for the $\beta$-alanine group (BA) and placebo group (PL).

\begin{tabular}{ccccc}
\hline Variable & Group & Pre-loading & Post-loading & Post-SIT \\
\hline TTE $(\mathrm{s})$ & BA & $166 \pm 27$ & $180 \pm 26^{\mathrm{ad}}$ & $210 \pm 23^{\mathrm{bce}}$ \\
& PL & $168 \pm 32$ & $170 \pm 32$ & $185 \pm 32^{\mathrm{bc}}$ \\
Anaerobic capacity $(\mathrm{kJ})$ & BA & $17.2 \pm 1.9$ & $17.9 \pm 2.1^{\mathrm{ad}}$ & $18.8 \pm 1.8^{\mathrm{bce}}$ \\
& PL & $17.3 \pm 2.4$ & $17.4 \pm 1.9$ & $17.7 \pm 2.3$ \\
$\mathrm{VO}_{2 \text { peak }}\left(\mathrm{L} \cdot \mathrm{min}^{-1}\right)$ & BA & $4.20 \pm 0.52$ & $4.22 \pm 0.61$ & $4.34 \pm 0.54^{\mathrm{bc}}$ \\
& PL & $4.31 \pm 0.60$ & $4.30 \pm 0.53$ & $4.44 \pm 0.45^{\mathrm{bc}}$ \\
Blood lactate $\left(\mathrm{mmol} \cdot \mathrm{L}^{-1}\right)$ & BA & $14.3 \pm 2.1$ & $15.5 \pm 2.4^{\mathrm{a}}$ & $16.1 \pm 2.5^{\mathrm{b}}$ \\
& PL & $14.6 \pm 2.2$ & $14.9 \pm 2.6$ & $15.6 \pm 2.9^{\mathrm{b}}$ \\
\hline
\end{tabular}

Note. $\overline{\mathrm{BA}}=\beta$-alanine group; $\mathrm{PL}=$ placebo group; $\mathrm{SIT}=$ sprint-interval training; $\mathrm{SEM}=$ standard error of the mean.

Significant differences within group; ${ }^{\mathrm{a}}$ post-loading vs. pre-loading, ${ }^{\mathrm{b}}$ post-SIT vs. pre-loading, cpost-SIT vs. post-loading.

Significant differences between groups; ${ }^{\mathrm{d}}$ post-loading vs. post-loading, ${ }^{\mathrm{e}}$ post-SIT vs. post-SIT 
Table 3 - Absolute change ( \pm 90\% confidence limits) in performance test results following 4 weeks of supplementation loading (post-loading) relative to pre-loading and following the 5 -wk sprint-interval training program (post-SIT) relative to post-loading for the $\beta$-alanine group (BA) and placebo group (PL).

\begin{tabular}{|c|c|c|c|c|c|c|}
\hline Variable & $\begin{array}{c}\Delta \mathbf{B A}-\Delta \mathbf{P L} \text { from } \\
\text { pre- to post- } \\
\text { loading } \\
\text { (Absolute } \\
\text { change } \pm \\
90 \% \mathrm{CL}) \\
\end{array}$ & $\begin{array}{c}\text { ANOVA } \\
\text { Group X Trial } \\
\text { interaction, } P \\
\text { value }\end{array}$ & $\begin{array}{c}\text { Magnitude- } \\
\text { based } \\
\text { inferences (BA } \\
\text { vs. PL; +/ /-) }\end{array}$ & $\begin{array}{c}\Delta \mathrm{BA}-\Delta \mathrm{PL} \text { from } \\
\text { post-loading to } \\
\text { post-SIT } \\
\text { (Absolute change } \\
\pm 90 \% \mathrm{CL} \text { ) }\end{array}$ & $\begin{array}{c}\text { ANOVA } \\
\text { Group X Trial } \\
\text { interaction, } P \\
\text { value }\end{array}$ & $\begin{array}{c}\text { Magnitude- } \\
\text { based } \\
\text { inferences (BA } \\
\text { vs. PL; +/ /-) }\end{array}$ \\
\hline $4 \times 1-\mathrm{km}$ & & & & $x_{1}$ & & \\
\hline Mean power output (W) & $12.5 \pm 9.7^{\mathrm{a}}$ & 0.040 & $73 / 27 / 0$ & $15.6 \pm 12.8^{\mathrm{a}}$ & 0.040 & $80 / 20 / 0$ \\
\hline \multicolumn{7}{|l|}{$4-\mathrm{km} \mathrm{TT}$} \\
\hline Mean power output (W) & $12.5 \pm 10.0^{\mathrm{a}}$ & 0.046 & $86 / 14 / 0$ & $8.2 \pm 12.0$ & 0.320 & $52 / 38 / 10$ \\
\hline Mean performance time (s) & $7.1 \pm 5.7^{\mathrm{a}}$ & 0.040 & $87 / 13 / 0$ & $-3.6 \pm 6.0$ & 0.280 & $51 / 44 / 5$ \\
\hline \multicolumn{7}{|l|}{$10-\mathrm{km} \mathrm{TT}$} \\
\hline Mean power output (W) & $1.8 \pm 2.4$ & 0.520 & $0 / 100 / 0$ & $-0.1 \pm 2.6$ & 0.820 & $0 / 100 / 0$ \\
\hline Mean performance time (s) & $-3.5 \pm 6.7$ & 0.284 & $9 / 91 / 0$ & $0.6 \pm 12.0$ & 0.930 & 4 / 95 / 1 \\
\hline
\end{tabular}

Note. $\mathrm{BA}=\beta$-alanine group; $\mathrm{PL}=$ placebo group; $\mathrm{CL}=$ confidence limits; magnitude-based inferences: $+/ \sim /$ - = beneficial/trivial/harmful effect

${ }^{a}$ Denotes a significant difference between groups 